\title{
Telas de sombreamento na produção de morangueiro em ambiente protegido
}

\author{
Rosiani C da Costa ${ }^{1}$; Eunice O Calvete ${ }^{1}$; Flávio Henrique Reginatto²; Dileta Cecchetti ${ }^{1}$; Joana Tereza \\ Loss $^{1}$; Aline Rambo' ${ }^{1}$ Fernando Tessaro ${ }^{1}$ \\ ${ }^{1}$ UPF-FAMV, C. Postal 611, 99001-970 Passo Fundo-RS; 2UFSC-Fac. Ciências Farmacêuticas, Trindade, 88040-900 Florianópolis-SC; \\ rosianicastoldi@yahoo.com.br; calveteu@upf.tche.br; cecchetti@upf.br; 74515@upf.br; 80908@upf.br; ftessaro@pop.com.br; \\ freginatto@hotmail.com
}

\section{RESUMO}

Analisou-se a produção de duas cultivares de morangueiro, conduzido sob diferentes telas de sombreamento (coberturas) em ambiente protegido. O experimento foi realizado em estufa agrícola de aço galvanizado, com teto semicircular, coberta com filme de polietileno de baixa densidade (PEBD) de $150 \mu \mathrm{m}$ de espessura, no Setor de Horticultura da Universidade de Passo Fundo, RS. Os tratamentos constaram de um fatorial duplo com duas cultivares (Camarosa e Oso Grande) e 4 coberturas (testemunha sem tela e telas termo-refletora metálica, azul e vermelha com $40 \%$ de sombreamento) que foram distribuídas em delineamento de blocos casualizados, com 3 repetições com 20 plantas por parcela em área útil de $3 \mathrm{~m}^{2}$. Foram determinados o número total e comercial de frutos e a massa fresca total e comercial e de frutos deformados por planta. Monitorou-se a temperatura do ar e a radiação fotossinteticamente ativa (RFA), sendo essa verificada em dois dias típicos (dia ensolarado e dia nublado). Considerando as médias obtidas sob a ausência e presença de telas e o total das colheitas, as produções das cultivares Oso Grande e Camarosa foram semelhantes. Na tela azul essas cultivares apresentaram a menor massa fresca.

Palavras-chave: Fragaria x ananassa Duch, Aluminet ${ }^{\circledR}$, ChromatiNet $^{\circledR}$ azul e vermelha, número de frutos, competição de cultivares.

\begin{abstract}
Shading screens in the production of strawberry in greenhouse

The production of two strawberry cultivars achieved under different shading screens (covers) in protected environment was evaluated. The experiment was set up in galvanized steel closed greenhouse with semicircular ceiling covered with low-density polyethylene (LDPE) films $150 \mu \mathrm{m}$ thickness, in the University of Passo Fundo, Rio Grande do Sul State, Brazil. The treatments consisted of a double factorial with two cultivars (Camarosa and Oso Grande) and four covers (control without screen and metalic, blue and red thermo-reflective screens with $40 \%$ shading) which were distributed in randomized blocks lining with three repetitions with 20 plants per plot in an useful area of $3 \mathrm{~m}^{2}$. The total and commercial number of fruits and the total and commercial fresh mass of deformed fruits per plant were determined. Also, the air temperature and the photossintetically active radiation (PAR) were monitored, being verified in two typical days (sunny and cloudy day). Considering the averages obtained under the absence and presence of screens and the total of the crops the production of the cvs. Oso Grande and Camarosa were similar. Under the blue screen these cultivars showed the lowest fresh mass.
\end{abstract}

Keywords: Fragaria x ananassa Duch, Aluminet ${ }^{\circledR}$, blue chromatiNet $^{\circledR}$ and red chromatiNet ${ }^{\circledR}$, number of fruits, competition of cultivars.

(Recebido para publicação em 15 de julho de 2009; aceito em 2 de setembro de 2010) (Received on July 15, 2009; accepted on September 2, 2010)

\begin{abstract}
$\mathrm{A}$ produção do morangueiro compreende nove estádios de desenvolvimento (Antunes et al., 2006) sendo que, a transição da fase vegetativa para reprodutiva inclui a indução, iniciação, diferenciação e desenvolvimento floral (Antunes et al., 2006; Kirschbaum, 1998). Em geral, a indução floral é controlada pelo genótipo, fotoperíodo e temperatura, especialmente em genótipos de dias curtos. Kirschbaum (1998) concluiu que o florescimento está relacionado com fotoperíodo entre 8 e 14 horas e intervalos de temperaturas de $18-25^{\circ} \mathrm{C}$ (dia) e $9-16^{\circ} \mathrm{C}$ (noite). Além disto, este autor concluiu que baixa
\end{abstract}

intensidade de luz vermelha induz o florescimento sob fotoperíodos curtos e temperatura de $15^{\circ} \mathrm{C}$. Desta forma, a produção de flores e frutos no inverno pode ser melhorada pelo aumento da intensidade de luz nos níveis de 400 a $450 \mu \mathrm{mol} \mathrm{m} \mathrm{m}^{-2} \mathrm{~s}^{-1}$, incluindo a luz na faixa do espectro vermelho e melhorando a eficiência na RFA (Radiação Fotossinteticamente Ativa).

No Brasil, as principais regiões produtoras localizam-se em áreas de clima subtropical de altitude elevada, com temperatura amena. No final da década de 90 a cultura se expandiu para áreas de clima tropical de altitude média (Conti et al., 2002). A produção brasileira de frutos de morango chega a 37,6 mil toneladas, sendo esta última obtida em uma área estimada de 3,5 mil hectares, com destaque para os estados de Minas Gerais (41,4\%), Rio Grande do Sul $(25,6 \%)$ e São Paulo $(15,4 \%)$ (IEA, 2008). A produção no Rio Grande do Sul elevou-se de $2 \mathrm{t} \mathrm{ha}^{-1}$ para 60-70 t ha ${ }^{-1}$ devido à alta tecnologia (Pagot \& Hoffmann, 2003). O aumento da demanda, aliada à necessidade de produzir o ano todo destaca o cultivo protegido como boa alternativa.

$\mathrm{O}$ uso do ambiente protegido no cultivo do morangueiro possibilita 
vantagens comparado com o campo, pois além de proteger a cultura de ventos, granizos, chuvas, geadas e baixas temperaturas, minimiza o ataque de pragas e doenças, proporcionando melhores condições ao desenvolvimento da planta, aumentando a frutificação total e a produção comercial (Antunes et al., 2007). Exemplos de mecanismos para melhorar o cultivo são as telas coloridas e refletoras. As telas de sombreamento no interior do ambiente protegido, sob o filme de polietileno, podem proporcionar condição microclimática apropriada para o desenvolvimento da cultivar, reduzindo, principalmente, os efeitos nocivos da alta taxa de incidência da radiação solar e da temperatura sob a planta (Shahak et al., 2004).

As telas devem apresentar boa ventilação para possibilitar o seu uso no verão, além de diminuir a temperatura e evitar danos tanto no sistema radicular quanto nas folhas. (Anglés, 2001). No inverno, esta ventilação reduz os problemas com Botrytis, melhora as condições de fecundação e minimiza a condensação suprimindo o gotejamento. Outra característica que as telas exercem no ambiente é a distribuição uniforme da radiação difundindo-a equitativamente.

Novas tecnologias na utilização de telas estão sendo empregadas em substituição às malhas de sombreamento de cor preta cujo objetivo principal é proteger as plantas da radiação. Esses materiais de polietileno de baixa densidade (PEBD) são de várias colorações (azul, vermelho, amarelo, cinza) com funções específicas na sua utilização. Por exemplo, a malha termo-refletora de alumínio promove boa ventilação, distribuição uniforme da luz e aporte máximo da luz difusa e da reflexão da radiação infravermelha, tanto para evitar o excesso da temperatura como para economizar energia (Huertas, 2006). Já as telas de coloração vermelha transferem mais a luz do espectro nas ondas vermelho e vermelho distante e difundem a luz que passa através da malha, sendo eficiente no desenvolvimento da planta (Li, 2006). As de coloração azul proporcionam luz do espectro em comprimento de onda de 440-490 nm, intensificando o fototropismo e a fotos- síntese (Rodrigues, 2002).

Com a finalidade de compreensão dessas novas técnicas, o objetivo foi analisar a produção de duas cultivares de morangueiro conduzido sob diferentes telas de sombreamento (coberturas), no interior de ambiente protegido.

\section{MATERIAL E MÉTODOS}

O experimento foi realizado de 06 de maio a 18 de dezembro de 2007, em ambiente protegido, no Setor de Horticultura da Universidade de Passo Fundo em Passo Fundo, RS (28 $15^{\prime} 41^{\prime}$ 'S; $52^{\circ} 24^{\prime} 45^{\prime \prime} \mathrm{W} ; 709 \mathrm{~m}$ de altitude). O clima da região é do tipo Cfa na classificação de Köeppen, isto é, temperado com chuvas bem distribuídas e verão quente. O Microclima apresenta temperaturas mínimas absolutas de $-4,0^{\circ} \mathrm{C}$, média das mínimas no inverno entre $6^{\circ}$ e $8^{\circ} \mathrm{C}$, com geadas de até 30 dias e, inclusive, neve em alguns anos, bem como anos com temperaturas máximas de 38 a $40^{\circ} \mathrm{C}$.

Os tratamentos constaram de um fatorial duplo com duas cultivares (Camarosa e Oso Grande), quatro coberturas (testemunha sem tela; telas vermelha e azul com $40 \%$ de sombreamento; tela termo-refletora metálica) e parcelas subdivididas no tempo, distribuídos em delineamento de blocos casualizados, com três repetições com 20 plantas por parcela em área útil de $3 \mathrm{~m}^{2}$.

$\mathrm{O}$ ambiente protegido utilizado para o desenvolvimento do trabalho foi uma estufa agrícola, em aço galvanizado, com teto semicircular e área de 510 $\mathrm{m}^{2}$, coberta com filme de polietileno de baixa densidade (PEBD) de $150 \mu \mathrm{m}$ de espessura, possuindo aditivo antiultravioleta e anti-gotejamento. A parte interna da estufa foi dividida em três compartimentos de $170 \mathrm{~m}^{2}$ com tela de coloração branca, sendo uma delas destinada ao experimento.

As mudas oriundas da cultura de meristema foram transplantadas (06 de maio) no espaçamento $0,3 \times 0,3 \mathrm{~m}$. As parcelas foram revestidas com filme de polietileno de baixa densidade de cor preta (mulching), com 30 micra de espessura. Após o revestimento do solo (30 dias após o transplante), foram instalados túneis sobre as cultivares de morangueiro, medindo $0,8 \mathrm{~m}$ de altura, 3,3 m de largura e 3,7 m de comprimento, cobertos com as telas vermelha, azul e termo-refletora metálica.

Utilizou-se o sistema de irrigação por gotejamento espaçado de $0,3 \mathrm{~m}$. Uma vez por semana foi realizada a fertirrigação na formulação descrita por Calvete et al. (2007). Durante o desenvolvimento do cultivo foi realizado tratamento fitossanitário com três aplicações dos fungicidas iprodione e azoxystrabin e duas de acaricida abamectina.

No período de agosto a dezembro de 2007 foram monitoradas as temperaturas máximas e mínimas do ar através de termômetros localizados no interior das coberturas a $40 \mathrm{~cm}$ de altura do solo. A radiação fotossinteticamente ativa (RFA) foi verificada nos dias 25 de julho de 2007 (dia ensolarado) e 11 de setembro de 2007 (dia nublado), no período das 8:00 às 17:00 horas utilizando aparelho Quantum/radiometer/photometer LI-COR-LI 250, posicionado sob cada cobertura, na altura da planta $(0,2 \mathrm{~m})$.

Semanalmente foram determinados o número (total e comercial) e massa fresca $(\mathrm{g})$ total e de frutos comerciais e deformados em seis plantas centrais de cada parcela, seguindo a classificação adotada pela CEAGESP (2002). Os dados de quatro semanas, dessas variáveis, foram agrupados perfazendo cinco avaliações, sendo analisados como parcelas subdivididas no tempo. Os resultados foram submetidos à análise de variância e, quando ocorreu significância, as médias foram comparadas pelo teste de Tukey a 5\% de significância utilizando o programa estatístico SISVAR.

\section{RESULTADOS E DISCUSSÃO}

O número total de frutos não apresentou significância para diferenciar a interação tripla entre coberturas $\mathrm{x}$ cultivares $\mathrm{x}$ colheitas. Entretanto, ocorreu efeito das coberturas no cultivo do morangueiro nas diferentes épocas de colheita, como também das cultivares, independente das coberturas, entre as colheitas (Tabela 1). Não houve efeito de coberturas nas cultivares.

O número médio total de frutos por 
Tabela 1. Frutos totais e comerciais por planta $\left(n^{0}\right)$ de duas cultivares de morangueiro, em diferentes coberturas e em cinco épocas de colheita (total and commercial number of fruits of two strawberry cultivars in different shading coverages and in five harvest times). Passo Fundo, UPF, 2007.

\begin{tabular}{|c|c|c|c|c|c|c|c|}
\hline \multirow{3}{*}{$\begin{array}{l}\text { Coberturas (telas de } \\
\text { sombreamento) }\end{array}$} & \multicolumn{7}{|c|}{ Frutos/planta $\left(n^{0}\right)$} \\
\hline & \multicolumn{6}{|c|}{ Total } & \multirow{2}{*}{ Comercial } \\
\hline & Ago & Set & Out & Nov & Dez & Média & \\
\hline Testemunha & $0,2 \mathrm{aC}$ & $12,5 \mathrm{aC}$ & $41,8 \mathrm{aA}$ & $57,2 \mathrm{aB}$ & $103,0 \mathrm{aA}$ & $42,9 \mathrm{a}$ & $27,9 \mathrm{a}$ \\
\hline Vemelha & $0,7 \mathrm{aC}$ & $12,7 \mathrm{aC}$ & $36,7 \mathrm{aB}$ & $39,8 \mathrm{bB}$ & $77,7 \mathrm{bA}$ & $33,5 b$ & $26,5 \mathrm{a}$ \\
\hline Azul & $0,3 \mathrm{aC}$ & $15,3 \mathrm{aBC}$ & $28,3 \mathrm{aAB}$ & $35,3 \mathrm{bA}$ & $43,5 \mathrm{cA}$ & $24,5 b$ & $17,8 \mathrm{~b}$ \\
\hline Metálica & $0,0 \mathrm{aC}$ & $10,5 \mathrm{aC}$ & $33,8 \mathrm{aA}$ & $39,2 \mathrm{cA}$ & $67,7 \mathrm{bA}$ & $30,2 b$ & $23,5 \mathrm{ab}$ \\
\hline \multicolumn{8}{|l|}{ Cultivares } \\
\hline Camarosa & $0,5 \mathrm{aC}$ & $11,9 \mathrm{aC}$ & $39,9 \mathrm{aB}$ & $39,8 \mathrm{bB}$ & $81,8 \mathrm{aA}$ & $34,8 \mathrm{a}$ & $24,6 \mathrm{a}$ \\
\hline Oso Grande & $0,1 \mathrm{aE}$ & $13,6 \mathrm{aD}$ & $30,4 \mathrm{aC}$ & $46,0 \mathrm{aB}$ & $64,0 \mathrm{bA}$ & $30,8 \mathrm{a}$ & $23,2 \mathrm{a}$ \\
\hline Média & 0,3 & 12,7 & 35,1 & 42,8 & 72,9 & 32,8 & 23,9 \\
\hline CV Coberturas (\%) & & & & 41,5 & & & \\
\hline CV Cultivares (\%) & & & & 37,3 & & & \\
\hline
\end{tabular}

Médias seguidas de mesmas letras minúsculas na coluna e maiúsculas na linha não diferem entre si pelo teste de Tukey a $5 \%$ de significância (means followed by same small letters in the column and capital letters in the line do not differ among themselves by Tukey test, $\mathrm{p}<0.05$ ).

Tabela 2. Massa fresca de frutos de morangueiro, em diferentes coberturas e em cinco épocas de colheita (fresh mass of strawberry fruits under different coverages and in five harvest times). Passo Fundo, UPF, 2007.

\begin{tabular}{lccc}
\hline \multirow{2}{*}{$\begin{array}{c}\text { Coberturas } \\
\text { (telas de sombreamento) }\end{array}$} & Total & Comercial & Deformados \\
\cline { 2 - 4 } Testemunha & $357,2 \mathrm{a}$ & $287,1 \mathrm{a}$ & $168,8 \mathrm{a}$ \\
Vemelha & $319,9 \mathrm{a}$ & $287,6 \mathrm{a}$ & $142,2 \mathrm{ab}$ \\
Azul & $224,0 \mathrm{~b}$ & $195,9 \mathrm{~b}$ & $96,6 \mathrm{~b}$ \\
Metálica & $289,9 \mathrm{ab}$ & $260,2 \mathrm{ab}$ & $134,6 \mathrm{ab}$ \\
\hline Cultivares & \multicolumn{3}{c}{} \\
\hline Camarosa & $304,6 \mathrm{a}$ & $259,8 \mathrm{a}$ & $150,0 \mathrm{a}$ \\
Oso Grande & $290,9 \mathrm{a}$ & $255,6 \mathrm{a}$ & $120,1 \mathrm{a}$ \\
\hline Colheitas & \multicolumn{3}{c}{} \\
\hline Ago & $3,7 \mathrm{c}$ & $3,7 \mathrm{~b}$ & $1,2 \mathrm{c}$ \\
Set & $127,6 \mathrm{c}$ & $113,2 \mathrm{~b}$ & $59,0 \mathrm{bc}$ \\
Out & $391,6 \mathrm{~b}$ & $367,5 \mathrm{a}$ & $233,6 \mathrm{a}$ \\
Nov & $395,2 \mathrm{~b}$ & $321,3 \mathrm{a}$ & $159,9 \mathrm{ab}$ \\
Dez & $570,5 \mathrm{a}$ & $482,8 \mathrm{a}$ & $221,6 \mathrm{a}$ \\
\hline CV (\%) Cobertura & 44,5 & & \\
CV(\%) Cultivares & 37,6 & & \\
CV(\%) Meses & 37,8 & & \\
\hline Medinyyy & & & \\
\hline
\end{tabular}

Médias seguidas de mesmas letras na coluna não diferem entre si pelo teste de Tukey a 5\% de significância (means followed by the same letters in the column do not differ among themselves by Tukey test, $\mathrm{p}<0.05)$.

planta conduzida sem cobertura para as duas cultivares avaliadas foi superior estatisticamente aos tratamentos com cobertura (Tabela 1). A formação de frutos (número) por planta foi incrementada em todos os tratamentos, com a proximidade do verão (novembro e dezembro). Até essa época (outubro) não se observaram diferenças no número de frutos, em relação à presença ou não das coberturas. Considerando cada cultivar independente das coberturas ao longo das colheitas, verificou-se que as cultivares Camarosa $(81,8)$ e Oso
Grande $(64,0)$ obtiveram maior número de frutos no mês de dezembro (Tabela 1), onde cv. Camarosa foi superior a cultivar Oso Grande. Esse incremento e as diferenças no número total de frutos com a proximidade do verão (novembro e dezembro) poderiam estar associados ao aumento da temperatura (Figura 1A) e provavelmente a radiação fotossinteticamente ativa.

Com relação ao número médio de frutos comercializáveis, não houve efeito positivo para as interações triplas e duplas. Entretanto, houve significância isoladamente entre as coberturas (Tabela 1). Independente da cultivar e considerando a média de todas as colheitas, o maior número de frutos comerciais foi obtido no tratamento sem cobertura $(27,9)$ e sob a tela vermelha $(26,5)$, os quais não diferiram da cobertura com tela metálica $(23,5)$. Já as plantas sob a tela azul apresentaram resultados inferiores às demais $(17,8)$, porém semelhantes às obtidas na tela metálica. A provável explicação parece estar na intensidade de radiação fotossinteticamente ativa (RFA) obtida sob as coberturas (Figuras 1B e 1C). Em dia ensolarado, a densidade da radiação RFA sob as coberturas azuis $\left(213,43\right.$ a $\left.176,70 \mu \mathrm{mol} \mathrm{m}^{-2} \mathrm{~s}^{-1}\right) \mathrm{e}$ metálicas $\left(234,50\right.$ a $\left.223,7 \mu \mathrm{mol} \mathrm{m} \mathrm{m}^{-2} \mathrm{~s}^{-1}\right)$ foram semelhantes (Figura 1B). Já em dia nublado a intensidade da RFA, sob a cobertura azul, foi inferior às demais 


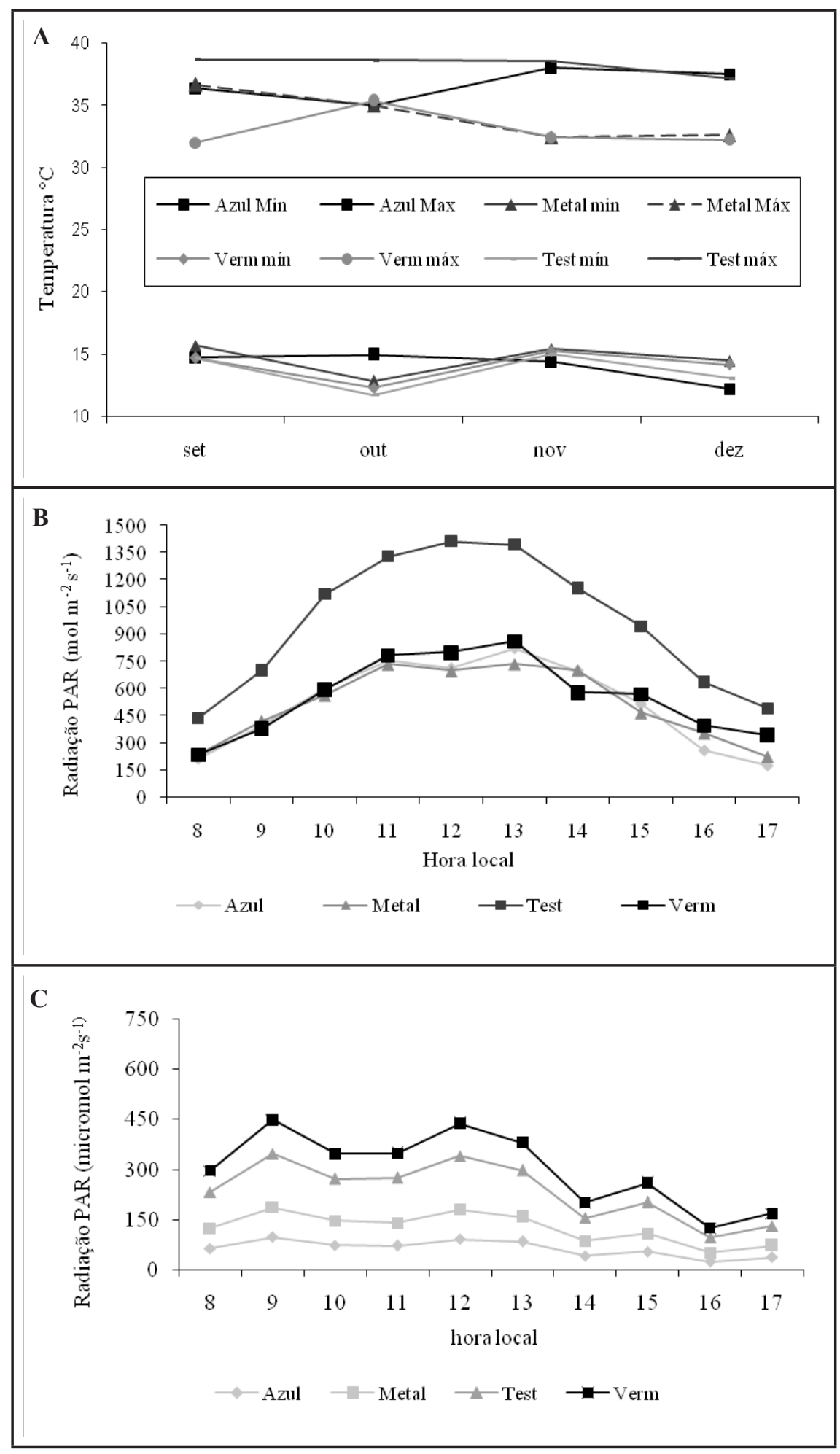

Figura 1. Temperaturas máximas e mínimas (A) durante o período de colheita e radiação fotossinteticamente ativa (RFA) em dia ensolarado (B), (25/07/2007) e dia nublado (C), $(11 / 09 / 2007)$ [maximum and minimum temperatures $(\mathrm{A})$ during the harvest period and the photosynthetically active radiation (PAR) in sunny day (B), (25/07/2007) and cloudy day (C), (11/09/2007)]. Passo Fundo, UPF, 2007.

coberturas no período compreendido entre 8:00 e 17:00 horas (Figura 1C). Estes resultados são concordantes com torando a RFA no período das 9:00 às 17:00 h, obtendo menor densidade da radiação fotossinteticamente ativa sob a tela azul $\left(28,99 \mathrm{Wm}^{-2}\right.$ a $\left.11,52 \mathrm{Wm}^{-2}\right)$.

As telas de sombreamento, que diminuem a intensidade luminosa no interior de ambiente protegido, afetam significativamente alguns comprimentos de onda, principalmente na faixa do azul (Kittas et al., 1999). Isso está de acordo com os resultados de produção de frutos comerciais, inferior com a cobertura azul em relação aos outros tratamentos, podendo estar relacionado às alterações espectrais causadas pelo material de cobertura (Tabela 1). Segundo OrenShamir et al. (2001), o espectro típico da tela azul apresenta um pico principal de transmitância na região do azul-verde (400-540 nm).

A exposição à luz vermelha e vermelha distante durante o crescimento e desenvolvimento foliar influencia o desenvolvimento de cloroplastos para garantir sobrevivência mais eficiente à planta (Kasperbauer \& Hamilton, 1984), o que, pode ter influenciado no aumento da capacidade fotossintética das plantas sem cobertura e com cobertura vermelha, quando comparadas à azul e à metálica. Isso ocorre devido à transferência de luz do espectro na faixa de ondas vermelha e vermelha distante ser maior promovendo melhor qualidade de luz e luz difusa e maior desenvolvimento vegetativo, enraizamento e produção (Li, 2006).

A massa fresca de frutos totais, comerciais e deformados nas diferentes coberturas, cultivares e épocas de colheita não apresentaram diferenças significativas na interação tripla e dupla. No entanto, houve diferenças significativas para cada tratamento isolado (Tabela 2).

As plantas das cultivares sem cobertura e com cobertura vermelha apresentaram maior massa fresca média total de frutos, não diferindo da cobertura metálica $(289,9$ g). Resultado semelhante foi obtido para a massa fresca comercial que produziu $287,1 \mathrm{~g}, 287,6$ g e 260,2 g por planta, respectivamente, para as cultivares sem cobertura e com cobertura vermelha e metálica. A menor massa fresca por planta total e comercial das cultivares avaliadas foi produzida 
sob a cobertura azul (Tabela 2).

As cultivares não apresentaram diferenças para massa fresca total, comercial e deformados de frutos por planta. A massa fresca total de fruto por planta foi maior no mês de dezembro $(570,5 \mathrm{~g}) \mathrm{em}$ relação aos demais meses de colheita. A massa fresca de frutos comerciais aumentou a partir de outubro e não foi significativamente diferente da massa fresca obtida em novembro e outubro.

A testemunha apresentou maior quantidade de frutos deformados que a cobertura azul, assim como nos meses de outubro e dezembro, os quais não diferiram de setembro (Tabela 2).

Analisando as épocas de colheita (Tabelas 1 e 2) constatou-se aumento no número e na massa fresca total de frutos, a partir do início da colheita (agosto) até o final (dezembro). Esses resultados não condizem com cultivares de dias curtos (florescem em fotoperíodo menor que 14 horas de luz e em temperaturas entre 8 e $15^{\circ} \mathrm{C}$ ), quando o pico de produção ocorre em meados do mês de outubro (Oliveira et al, 2008). Agricultores da região do Planalto do Rio Grande do Sul relataram características de produção, em 2007, similares às obtidas nesta pesquisa, sendo produções inferiores a anos anteriores em ambiente protegido. Essa informação confirma os resultados obtidos, nesse experimento, com média de 304,6 g por planta na cv. Camarosa e 290,9 g na Oso Grande, sendo inferiores aos encontrados por Oliveira et al. (2008), Calvete et al. (2008) e Antunes et al. (2007).

Com base nos resultados deste experimento as cvs. Camarosa e Oso Grande de morangueiro, produzidas em ambiente protegido no ano de 2007, incrementam a produção a partir de outubro, sendo crescente até o final de dezembro. Considerando as médias obtidas sob a ausência e presença de telas e o total das colheitas as produções das cultivares Oso Grande e Camarosa são semelhantes. Na tela azul essas cultivares apresentam a menor massa fresca.

\section{AGRADECIMENTOS}

À CAPES pela concessão da bolsa de estudos à acadêmica de mestrado Rosiani Castoldi da Costa e ao CNPq e FAPERGS, pelas bolsas de Iniciação científica aos alunos de Agronomia Fernando Tessaro, Aline Rambo e Joana Teresa Loss, respectivamente.

\section{REFERÊNCIAS}

ANGLÉS M. 2001. Control climático y ciclo de cultivo. Horticultura 152: 1-7.

ANTUNES OT; CALVETE EO; ROCHA HC; NIENOW AA; MARIANI F; WESP CL. 2006. Floração, frutificação e maturação de frutos de morangueiro cultivados em ambiente protegido. Horticultura Brasileira 24: 426-430.

ANTUNES OT; CALVETE EO; ROCHA C; NIENOW AA; CECCHETTI D; RIVA MR. 2007. Produção de cultivares de morangueiro polinizadas pela abelha jataí em ambiente protegido. Horticultura Brasileira 25: 60-65.

CEAGESP. 2002. Normas de classificação do morango. São Paulo: CQH/CEAGESP, 6p.

CALVETE EO; NIENOW AA; WESP CL; CESTONARO L; MARIANI F; FIOREZE I; CECCHETTI D; CASTILHOS T. 2007. Produção hidropônica de morangueiro em sistema de colunas verticais, sob cultivo protegido Revista Brasileira de Fruticultura 29: 524-529.

CALVETE EO; MARIANI F; WESP CL; NIENOW AA; CASTILHOS T; CECCHETTI D. 2008. Fenologia, produção e teor de antocianinas de cultivares de morangueiro em ambiente protegido Revista Brasileira de Fruticultura 30: 396-401.

CONTI JH; MINAMI K; TAVARES FCA. 2002. Produção e qualidade de frutos de morango em ensaios conduzidos em Atibaia e Piracicaba Horticultura Brasileira 20: 10-17.

HUERTAS L. 2006. Control ambiental em el vivero. Horticultura Internacional n. extra: $77-84$.

IEA-INSTITUTO DE ECONOMIAAGRÍCOLA.
2008. Pólos de produção do morango. Disponível em: www.iea.sp.gov.br/out/ vertexto.php?codtexto=11. Acessado em 11 outubro de 2008.

KASPERBAUER MJ; HAMILTON JL. 1984. Chloroplast structure and starch grain accumulation in leaves that received different red and far-red levels during development. Plant Physiology 74: 967-970.

KIRSCHBAUM DS. 1998. Temperature and growth regulator effects on growth and development of strawberry (Fragaria $x$ ananassa Duch.). Florida: University of Florida. 144p. (Tese mestrado).

KITTAS C; BAILLE A; GIAGLARAS P.1999. Influence of covering material and shading on the spectral distribution of light in greenhouse Journal of Agricultural Engineering Research 73: 341-351.

LEITE CA; ITO RM; GERALD LTC; GANELEVIN R; FAGNANI MA. 2008. Light spectrum management using colored nets aiming to controlling the growth and the blooming of Phalaenopsis sp. Disponível em: http://www.polysack.com/files/ e9f9f2aca300c62ae62d46141f287901.pdf. Acessado em: 10 de outrubro de 2008.

LI JC. 2006. Uso de mallas en invernaderos. Horticultura Internacional n. extra: 8691.

OLIVEIRA RP; SCIVITTARO WB; FINKENAUER D. 2008. Produção de morangueiro da cv. Camino Real em sistema de túnel Revista Brasileira de Fruticultura 30: 681-684.

OREN-SHAMIR M; GUSSAKOVSKY EE; SPIEGEL E; NISSIM-LEVI A; RATNER K; GILLER YE; SHAHAK Y.2001. Colored shade nets can improve the yield and quality of green decorative branches of Pittosporum variegatum. Journal Horticulture Science Biotechnology 76: 353-361.

PAGOT E; HOFFMANN A. 2003. Produção de pequenas frutas no Brasil. In: SEMINÁRIO BRASILEIRO SOBRE PEQUENAS FRUTAS, 1. 2003. Anais... Vacaria: Embrapa Uva e Vinho, 2003. p.9-17.

RODRIGUES LRF. 2002. Técnicas de cultivo hidropônico e de controle ambiental no manejo de pragas, doenças e nutrição vegetal em ambiente protegido Jaboticabal: Funep. $762 \mathrm{p}$.

SHAHAK Y; GUSSAKOVSKY EE; COHEN Y; LURIE S; STERN R; KFIR S; NAOR A; ATZMON I; DORON I. 2004. GreenblatAvronColorNets: a new approach for light manipulation in fruit trees. Acta Horticulturae 636: 609-616. 